\title{
Frequency bands in seeing and remembering: comparing ADHD and typically developing children
}

\section{Rosa Angela Fabio ${ }^{1}$ - Tindara Caprì ${ }^{1}$ \\ Nasrin Mohammadhasani ${ }^{2}$ - Antonio Gangemi ${ }^{1}$ \\ Antonella Gagliano ${ }^{3}$ - Gabriella Martino ${ }^{1}$}

${ }^{1}$ Cognitive Empowerment Laboratory, Department of Cognitive Sciences, Psychological, Educational and Cultural Studies, University of Messina, Messina, Italy

${ }^{2}$ Department of Humanities, University of Tarbiat Madares, Jalal Ale Ahmad Highway, Tehran, Iran

${ }^{3}$ Department of Pediatric and Biomedic Science, University Hospital "Gaetano Martino", Messina, Italy

\section{ABSTRACT}

This study aimed to examine whether the visual working memory (VWM) phases, encoding and recall, may be impaired in children with Attention Deficit/Hyperactivity Disorder (ADHD). The second aim was to measure electroencephalographic (EEG) correlates of two types of encoding: efficient and inefficient encoding. 23 children with $A D H D$, age and sex matched with 23 non-ADHD subjects, were recruited. With reference to neurophysiological measures, an EEG was recorded before the WMT, during the encoding and finally during the recalling phases of the WMT. With reference to neuropsychological measures, we tested the encoding phase through eye-tracker measures and the recalling phase through the free recall span measure. In comparison to the control subjects, the ADHD group showed lower eye-tracker measurements and free recall span. They also showed lower alpha, beta and theta levels than the control group. Only in the efficient encoding, the control and ADHD groups showed similar beta bands. These results suggest that the ADHD group showed a decrease in the power of nearly all bands. 
Keywords: Attention-Deficit/Hyperactivity Disorder; Children; EEG; Encoding; Frequency bands

\section{INTRODUCTION}

For more than 40 years, electroencephalography (EEG)-based research has attempted to refine and quantify the neurophysiological features of attention deficit/hyperactivity disorder (ADHD). An extensive range of studies in this area has been conducted and reviewed (Lenartowicz \& Loo, 2014). Based on reviews of current literature on ADHD, the most consistent EEG feature is elevated power of slow waves (4-7 Hz "theta") and/or decreased power of fast waves (14-30 Hz "beta"), typically recorded over fronto-central electrodes, which are sometimes combined and quantified by the theta/beta ratio (TBR) (Chabot \& Serfontein, 1996; Clarke, Barry, McCarthy, \& Selikowitz, 1998; Clarke, Barry, McCarthy, \& Selikowitz , 2001a; Guzmán et al., 2015; Lazzaro et al., 1998; Loo, et al., 2010; Ogrim, Kropotov, \& Hestad, 2012). However, recent research has not confirmed the findings for theta and TBR. Particularly, with reference to TBR, Loo, Cho, Hale, McGough, McCracken and Smalley (2013) found no significant differences between 562 children, adolescents, and adults with ADHD compared to 309 non-ADHD controls, although modest heterogeneity was attributed to ADHD subtype with psychiatric co-morbidity (Lenartowicz \& Loo, 2014). Furthermore, in a recent meta-analysis, Arns, Conners and Kraemer (2013) confirmed the lack of theta or TBR differences in children with ADHD versus typically developing (TD) subjects. Snyder and Hall (2006) also showed that increased TBR correlates strongly with agerelated changes in ADHD behavioural symptomatology over time. Therefore, it seems that elevated spectral power in the theta band or TBR is not a homogenous feature within ADHD, but characterizes only a subset of patients, in particular the combined subtype of ADHD (Lenartowicz \& Loo, 2014).

Aside from the findings for theta and TBR, results for other frequency bands, such as beta and alpha, have been even more variable. As regards alpha band activity, some studies have reported reduced alpha band power in ADHD subjects compared to normal controls, whereas others have found increased alpha activity or no significant differences (El-Sayed, Larsson, Persson, \& Rydelius, 2002; Clarke et al., 2011). Similarly, the findings for beta activity have been less consistent (Barry, Clarke, Johnstone, \& Brown, 2009; Clarke, Barry, McCarthy, \& Selikowitz, 2001b; Kamada et al., 2016). Although most research has shown relative decreased beta band power, several other studies 
have not confirmed this result. For instance, Kuperman, Johnson, Arndt, Lindgren, and Wolraich (1998) and Clarke et al. (2011) have found increased beta band power in ADHD subjects. In addition, a recent study has also revealed that the power of the beta band in the ADHD group was significantly higher in all regions of the brain, except for the occipital region, compared with control children (Kamida et al., 2016).

Summarizing the data presented above, there is no consensus on frequency bands in ADHD research: either theta, alpha and beta, individually (e.g., absolute or relative band power) or compared to each other (such as, theta/beta power or amplitude ratio). The underlying reasons may be ascribed to the heterogeneity of ADHD subtypes and of tasks.

With reference to clinical heterogeneity, children with ADHD show significant heterogeneity in behavioural, cognitive and neurobiological domains; consequently, they may also exhibit neurophysiological heterogeneity. A considerable number of EEG studies have demonstrated distinct EEG profiles within the ADHD population. Clarke et al. (2011) revealed five unique clusters within EEG data of 264 children with ADHD. Of these, only two clusters (55 participants, 36\% of the ADHD sample) showed elevated theta (and reduced beta), whereas the remaining three clusters were characterized by: elevated beta power $(12.5-25 \mathrm{~Hz})$, elevated slow wave power $(<12.5 \mathrm{~Hz})$ and elevated frontal alpha power $(7.5-12.5 \mathrm{~Hz})$. Therefore, considering the clinical heterogeneity of ADHD and the complexity of the information available from EEG signals, the neurophysiological heterogeneity reported in EEG literature on ADHD is not surprising.

With reference to the heterogeneity of tasks, it has been proposed that frequency bands can reflect task-related cortical activation (Loo \& Makeig, 2012). However, in considering the most examined frequency bands in children with ADHD, increased fronto-central theta band power has been recorded in both the resting state and during working memory (WM) task trials (Loo \& Makeig, 2012; McCarthy, Skokauskas, \& Frodl, 2014). Moreover, several studies have shown that alpha and beta activities may differ depending on age and ADHD subtype (Loo et al., 2010; Loo \& Makeig, 2012). Thus, frequency band activities can represent different phenomena depending on the recording circumstances, age of subjects and heterogeneity of ADHD.

To reduce the heterogeneity, it is also important to distinguish data on frequency bands in relation to the two phases of VWM: encoding and recalling. To our knowledge, no work has studied the differences of bands when the encoding is efficient and when the encoding is not efficient. Efficient encoding (EE) refers to the correspondence between the stimuli which the child looked at during the encoding phase and the retrieval of the same stimuli in the free recall phase, whereas inefficient encoding (IE) refers to the lack of correspondence 
between the stimuli the child looked at during the encoding phase and the retrieval of the same stimuli in the free recall phase. We assume that frequency bands data may become homogeneous when considering only EE.

With reference recalling data, neuropsychological data were quite consistent in ADHD subtypes. The studies of Geurts, Verte, Oosterlaan, Roeyers, and Sergeant (2005) and Hinshaw, Carte, Sami, Treuting and, Zupan (2002) found no or limited differences between ADHD-C (combined subtype) and ADHD-I (inattentive subtype) on a series of executive functioning measures, among which there were visual working memory tasks. In another study, Liebel and Nelson (2017) compared three groups, ADHD predominantly inattentive (ADHD-I) and combined (ADHD-C) and normal controls matched by age and IQ, on visual working memory, planning, cognitive flexibility, and verbal fluency. Executive function measurements, again, did not show significant differences between the ADHD-C and ADHDI subtypes. Martínez, Cortes, and Sevilla (2018) and Fabio and Caprì (2015, 2017) examining the visuo-spatial working memory found no differences

Despite the consistency of these neuropsychological data, conversely, the context- or state-dependency of EEG abnormalities in ADHD during these tasks has been little investigated. For example, the pattern of brain activation correlates in WM during memory tasks is still unclear (Chamberlain et al., 2011; Engelhardt, Nigg, Carr, \& Ferreira, 2008; Fabio, 2017; Fabio \& Caprì, 2015; Manly, Anderson, Nimmo-Smith, Turner, Watson, \& Robertson, 2001; Mohammad et al., 2016; Westerberg, Hirvikoski, Forssberg, \& Klingberg, 2004). A recent EEG study, which examined whether neural indices of WM maintenance differ between young adults with ADHD and their healthy peers, demonstrated that the ADHD group exhibited lower posterior alpha power at a trend level compared to their healthy peers (Liu, Glizer, Tannock, \& Woltering, 2015). In another recent study, no difference was found between children with ADHD and typically developing children regarding TBR, theta power or alpha and beta frequency bands, neither before nor after the execution of 3 n-back tasks (Buyck \& Wiersema, 2015).

Due to these different results in EEG parameters, it is important to identify the conditions under which WM impairments appear, in order to understand the clinical significance of such deficits in terms of the neural and cognitive mechanisms underlying WM dysfunction in ADHD. Therefore, EEG research that separates the phases of WM is required.

To our knowledge, two studies have investigated frequency bands during a WM task, considering multiple WM phases. Lenartowicz et al. (2014) examined the EEG correlates of spatial WM (SWM) deficits in ADHD during three task phases: vigilance, encoding and maintenance. Their results showed reduced alerting responses to fixation and enhanced group differences during 
encoding and maintenance (in the alpha band).

On the other hand, Gomarus, Wijers, Mindera, and Althaus (2009) measured event-related changes in scalp alpha and theta power while children with ADHD memorized a set of letters for later retrieval, and did not find differences in the alpha power band. However, there are methodological differences between the two studies. Gomarus, Wijers, Mindera, and Althaus (2009) used a verbal WM task and considered children with ADHD-C only, whereas Lenartowicz et al. (2014) employed an SWM task and included children with combined and inattentive type and children with comorbid oppositional defiance disorder (ODD) in the ADHD group.

Although these findings are important in terms of their clinical implications, a critical question regarding WM dysfunctions in ADHD and EEG correlates of WM component processes still remains unanswered. The issue is to determine to what extent the multiple processes of WM are differently impaired, in order to disentangle the neural correlates underlying WM processes in ADHD. In line with this logic, the main aims of the present study were: a) to examine whether the visual working memory (VWM) phases, encoding and retrieval, may be differentially impaired in children with ADHD and b) to define EEG correlates of VWM. In accordance with previous studies (Mohammadhasani, Fabio \& Caprì, in press; Mohammadhasani, Fabio, Fardanesh \& Hatami, 2015; Türkan, Amado, Ercan, \& Percinel, 2016; Wiegand, 2016), it was hypothesized that the ADHD group would show a shorter duration and lower frequency of fixation than TD subjects; moreover we awaited a poorer VWM performance in ADHD compared to the typically developing group. Secondly, with reference to controversial findings in EEG literature on ADHD, and based on the notion that there is an excess of beta activity in the EEG of children with ADHD (Clarke, Barry, McCarthy, \& Selikowitz, 2001; Lenartowicz \& Loo, 2014), it was hypothesized that the ADHD group would exhibit a different power in beta, theta and alpha bands compared to the TD group.

Furthermore, as above mentioned, in the present study, we also distinguished two types of encoding: efficient and inefficient. With reference to the different bands, it was hypothesized that the groups with ADHD would show the same performance as the control group in the efficient encoding process. 


\section{METHOD}

\subsection{Participants}

23 children with ADHD and 23 TD children participated in the study. The ADHD group was recruited and diagnosed at the outpatient clinic of the Department of Pediatric and Biomedical Science. The TD group was recruited through advertisement in local schools. Both groups were screened for the study through a clinical diagnosis carried out by one psychiatrist and one experienced neuropsychologist during individual interviews. After individual clinical assessment, the subjects without disorders identified by DSM-V (American Psychiatric Association, 2013), and who were diagnosed as not being affected by behavioural, emotional and/or relational problems, were included in this study.

Hence, of the 23 patients included in the study, 11 were classified as being of the inattentive subtype, and 12 of the combined subtype. None of the children with ADHD were taking medication; nor did they have a psychopathology associated with anxiety, depression, psychosis or intellectual disability. The control participants were diagnosed as not affected by behavioural, emotional, attentional and/or relational problems by the specialised psychologists.

The demographic characteristics for the two participant groups are summarised in Table 1.

Table 1. Demographic characteristics of the three groups participating in the experiment

\begin{tabular}{|c|c|c|}
\hline Groups & Measures & Values \\
\hline \multirow[t]{5}{*}{ ADHD $\quad-$ I } & $n$, boys/girls & $8 / 3$ \\
\hline & Age, $M(S D)$ & $9.80(4.80)$ \\
\hline & $\mathrm{IQ}, M(S D)$ & $95.00(6.50)$ \\
\hline & Distractibility, $M(S D)$ & $19.70(2.45)$ \\
\hline & Hyperactivity, $M(S D)$ & $3.30(3.01)$ \\
\hline \multirow[t]{5}{*}{ ADHD-C } & $n$, boys/girls & $8 / 4$ \\
\hline & Age, $M(S D)$ & $10.1(5.10)$ \\
\hline & $\mathrm{IQ}, M ;(S D)$ & $96.00(5.60)$ \\
\hline & Distractibility, $M(S D)$ & $18.80(2.50)$ \\
\hline & Hyperactivity, $M(S D)$ & $16.90(2.20)$ \\
\hline \multirow[t]{5}{*}{ Control } & $n$, boys/girls & $13 / 10$ \\
\hline & Age, $M(S D)$ & $10.5(6.53)$ \\
\hline & $\mathrm{IQ}, M(S D)$ & $107.50(7.95)$ \\
\hline & Distractibility, $M(S D)$ & $1.00(0.20)$ \\
\hline & Hyperactivity, $M(S D)$ & $7.0(0.31)$ \\
\hline
\end{tabular}

Neuropsychological Trends - 24/2018

http://www.ledonline.it/neuropsychologicaltrends/ 


\subsection{Materials and instruments}

To evaluate WM, the Free Recall subtest (computerized form) of the WMT was employed (Green, Allen, \& Astner, 1996) in the current study. The task involved learning 3 lists of 16 categorical unrelated words in an array of 4X4, which were presented on an eye tracker screen.

The WMT was presented through an eye tracking device, model «iAble( - MyTobii ${ }^{\circledR}$ («D10» version). Experiments were conducted in a screen resolution of $1024 \times 768$. The eye tracker was calibrated to each participant's left and right eyes before the experiment. A stabilized viewing position and distance were provided with a chin rest (distance from the screen was constantly $50 \mathrm{~cm}$ ). The eye tracker allowed definition of the areas of interest (AoI) within the stimuli chosen for statistical analysis of the eye tracking parameters. AoI clusters refer to selected specific areas that were used for recalling details of the images.

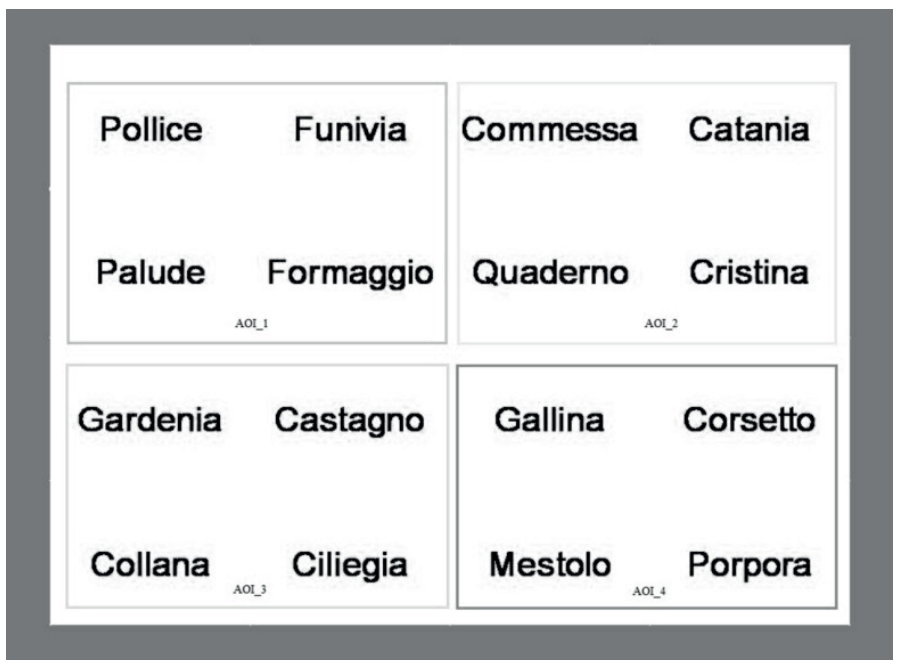

Figure 1. Areas of interest (AoI)

\subsection{EEG recording and analysis}

The EEG signal was acquired with Micromed instrumentation (Treviso, Italy) using an EEG amplifier and a pre-wired headphone consisting of 21 electrodes 
positioned on the scalp of the subjects, in accordance with parameters provided by the international 10/20 measurement system.

Signals were recorded with a sampling rate of $1024 \mathrm{~Hz}$, and already filtered in the acquisition phase through a band-pass anti-aliasing $\mathrm{AD}$ filter (0.15-269.5 $\mathrm{Hz}$ ). The sampling rate was $250 \mathrm{~Hz}$.

The reference electrode was placed in the $\mathrm{FPz}$ position and the ground electrode placed posteriorly to $\mathrm{Fz}$, as reported in previous studies (Formaggio et al., 2008; Storti, Formaggio, Beltramello, Fiaschi, \& Manganotti, 2010). The acquisition phase of each experimental session began with registration of the baseline: the subject remained seated with eyes open for 5 minutes during which time the EEG signal was measured. Regarding the EEG signal, it was seen that the main noise was due to network interference and located at $50 \mathrm{~Hz}$; it was also noted that the useful part of the signal was between 0 and $30 \mathrm{~Hz}$. Taking into account these characteristics of the EEG signal, it was decided to apply a low-pass FIR filter to the signal of each electrode with band pass $0-30 \mathrm{~Hz}$, transition band between 30 and $40 \mathrm{~Hz}$ and with bandwidth ripples equal to 0.01 , respectively. In addition to this filtering, the sample average was subtracted along with the elimination of any trends due to drift effect; for this operation, carried out prior to spectral analysis, the Matlab function detrend was used. The time span of the relative spectral power and the temporal coherence for the alpha $(7-12 \mathrm{~Hz})$, beta $(15-22 \mathrm{~Hz})$ e theta $(4-8 \mathrm{~Hz})$ frequency bands were studied. Spectral analysis was evaluated by transforming the signal from the time domain to the frequency domain using the Welch method (Welch, 1967).

\subsection{Procedure}

Participants were tested individually at the Department of Pediatric and Biomedical Science. The ADHD group and the TD group were tested separately. All children were tested in the morning from 9.00 to 12.00 a.m.

During the encoding phase, participants were asked to look for each of the 3 lists of 16 categorical unrelated words in 3 different arrays of the eye tracker screen. The children were instructed not to move during this phase.

During the recall phase, participants were asked to recall as many words as possible from the list of words immediately after the presentation of each list. Each list was presented individually for 1 minute, following a randomized order. After recall, the experimenter pressed the mouse to display the next list. Figure 2 shows the procedure. 


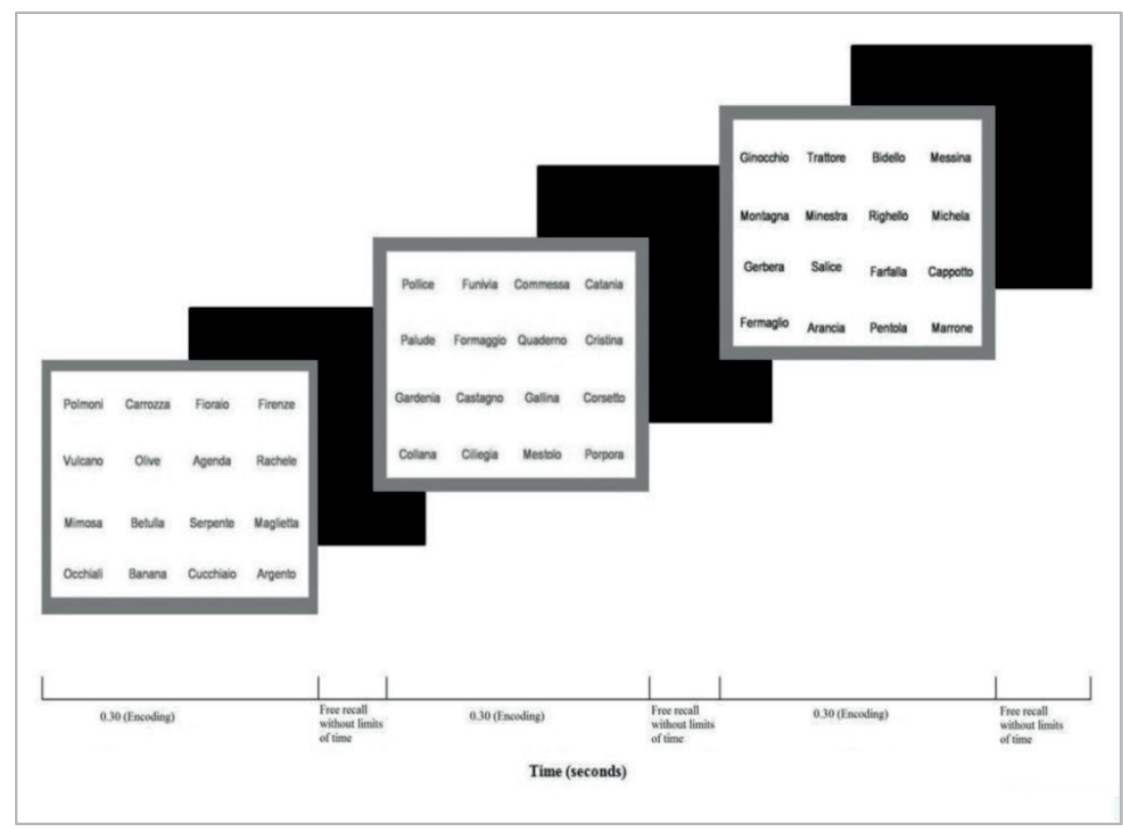

Figure 2. Procedure

\subsection{Measures}

With reference to the encoding phase the following parameters were computed: fixation length (FL), length of time (seconds) spent by the child looking at the words; fixation count (FC), the number of times the child looked at the words. Fixations were extracted using a threshold of $100 \mathrm{~ms}$.

As above mentioned, we categorized EE and IE. EE was coded when the child looked at a word for more than five times (predetermined by the heatmap of the eye tracker software that become orange coloured with that intensity) during the encoding phase and he retrieves the same words in the free recall phase, whereas IE was coded when there was a lack of correspondence between the words the child looked at more than five times during the encoding phase and the retrieval of the same words in the free recall phase. To separate efficient and inefficient encoding, we selected two set of 5 words in each task and for each subjects. These two set refer to those words in which there was presence or absence of correspondence between the stimuli the child looked at during the 
encoding phase and the retrieval of the same stimuli in the free recall.

With reference to the recall phase, the parameter was the number of correctly remembered words (CRW).

EEG neural responses were registered for all the time of the task during the baseline (before the WMT), during the encoding phase and the recall phase of the WM.

\subsection{Design}

With reference to the parameters of encoding and recalling, this study employed several one-way designs assuming Group as between-subjects independent (groups: ADHD-I, ADHD-C and TD subjects) and the different measures as dependent variables.

With reference to neurophysiological parameters, this study employs a $3 \mathrm{X}$ 2 factorial design, assuming groups and encoding (EE and IE) as independent variables and the different frequency bands as dependent variables.

\subsection{Statistical analyses}

The data were analysed using SPSS Version 14.0 for Windows. The descriptive statistics of the dependent variables were tabulated and examined. The alpha level was set to 0.05 for all statistical tests. In the case of significant effects, the effect size of the test was reported. We confirmed that these data were normally distributed using Shapiro-Wilk test before running the statistical analysis. All results are presented as means \pm standard deviations (SD). The effect sizes were computed and categorized according to Cohen (1988). Initially, the dependent variables listed above were considered during data analysis. Subsequently, statistical analysis focused also on the difference between EE and IE.

\section{RESULTS}

We evaluated the performance of the two clinical groups and the control group in two phases: encoding and free recall of the stimuli. Simultaneously, beta, alpha and theta frequency band activity was measured in all three groups. As written above, the encoding phase was measured through the FL and FC parameters while the recalling phase was measured through the CRW parameter.

With reference to the encoding phase, in relation to the parameters FC and FL we conducted two one-way AN.O.Vas assuming Group as independent variable. Group factor shows significant effects, $F(2,43)=14.36, \mathrm{p}<.001, \mathrm{~d}=0.90$ and $\mathrm{F}(2,43)=11.60$, 
$\mathrm{p}<.001, \mathrm{~d}=0.90$ respectively. This result indicates that FC and FL indices are lower in both $\mathrm{ADHD}$ groups than in the TD group. These two parameters (FC and FL) consistently show that subjects with ADHD look at the stimulus words which appear on the monitor fewer times and for a shorter duration of time than the TD group (Table 2).

With reference to the recalling phase, in relation to the parameter number of correctly CRW, we conducted a one-way AN.O.Va. assuming Group as independent variable. Group factor shows significant effects, $F(2,43)=11.02, p<.001, d=0.90$. Subjects with ADHD recall significantly less words than the TD group (Table 2).

Table 2. Means and (Standard Deviation) of the parameters

\begin{tabular}{lccc}
\hline Parameters & ADHD-I & ADHD-C & Control \\
\hline Number of correct words & $1.72(1.34)$ & $1.78(0.89)$ & $5.33(0.35)$ \\
FL & $19.85(5.34)$ & $12.10(7.69)$ & $22.30(0.96)$ \\
FC & $40.77(22.67)$ & $43.81(21.13)$ & $74.33(5.27)$ \\
\hline
\end{tabular}

With regard to neurophysiological parameters, statistical analyses were carried out on beta, theta and alpha bands. Table 3 shows the means and standard deviations for beta, alpha and theta power bands in the three groups.

With reference to the beta frequency band, the values were higher in TD group compared to the two clinical groups, $\mathrm{F}(1,43)=91.29, \mathrm{p}<.01, \mathrm{~d}=0.73$, and this result is already present in literature (Clarke, Barry, McCarthy, \& Selikowitz, 2001a, 2001b; El-Sayed, Larsson, Persson, \& Rydelius, 2002; Loo et al., 2010).

Table 3. Means and (Standard Deviation) for beta, alpha and theta power bands

\begin{tabular}{cccc}
\hline Bands & ADHD-I & ADHD-C & TD \\
\hline Beta EE & $15.36(0.35)$ & $15.38(0.48)$ & $17.00(0.56)$ \\
Beta IE & $14.73(0.28)$ & $14.24(0.38)$ & $15.73(0.39)$ \\
Theta EE & $6.51(0.42)$ & $6.36(0.90)$ & $7.82(0.12)$ \\
Theta IE & $6.42(0.37)$ & $6.17(0.88)$ & $7.73(0.09)$ \\
Alpha EE & $8.27(0.48)$ & $8.42(0.39)$ & $9.80(0.28)$ \\
Alpha IE & $8.15(0.42$ & $8.38(0.39)$ & $9.50(0.70)$ \\
$\begin{array}{c}\text { Differences between } \\
\text { Beta baseline and beta EE } \\
\text { Differences between }\end{array}$ & $1.82(0.34)$ & $1.40(0.60)$ & $1.20(0.35)$ \\
Beta baseline and beta IE & $0.08(0.32)$ & $0.12(0.50)$ & $0.51(0.32)$ \\
\hline
\end{tabular}

Since baseline values of the TD group showed higher indices of beta $F(1,43)=$ 
8.73, $\mathrm{p}<.01, \mathrm{~d}=0.73$, to check the hypothesis if there were group differences between the baseline and the phase of efficient encoding, the differences between the baseline beta index (BBI) and the beta index during the efficient encoding task (TBI) was calculated for each subject. This allowed to eliminate possible effects due to baseline level. What emerges, as can be seen in Table 3, is that beta activation is higher in the clinical groups than in the TD group; this result is not surprising given that the beta baseline was already higher in the TD from baseline phase.

Regarding the difference between EE and IE in relation to the increase in beta band, the Groups variable shows significant effects, $\mathrm{F}(2,43)=18.81, \mathrm{p}<.001 ; \mathrm{d}=$ 0.90 ; the interaction groups $\mathrm{x}$ type of encoding shows significant effects, $\mathrm{F}(2,43)=$ $5.10, \mathrm{p}<.01 ; \mathrm{d}=0.73$. This result indicates that both ADHD groups show a similar increase in beta when encoding is efficient, whereas they show significantly different activation when encoding is inefficient.

In summary, these results suggest that beta bands are higher in the TD group from the baseline phase. On the other hand, calculating the differences in beta between baseline and the level during the EE task, the increases were exactly the same in the ADHD-C group and the TD group, whereas there were differences in beta band increase when encoding was inefficient. Therefore, it is reasonable to suggest that when the ADHD-C group was adequately activated, the beta band neurophysiological profile was similar to that of the TD group.

With reference to theta band activity (Table 3 ), the TD group shows higher theta band power indices than the two clinical groups $\mathrm{F}(2,43)=4: 36, \mathrm{p}<.05 ; \mathrm{d}=0.78$. This result indicates that the two clinical groups have lower indices of theta, and this occurs in both EE and IE. Finally, regarding the alpha band (Table 3), there are significant differences, both at baseline, $\mathrm{F}(2,43)=9.92, \mathrm{p}<.05 ; \mathrm{d}=0.78$ and after the task, $\mathrm{F}(2,243=6.25, \mathrm{p}<.02 ; \mathrm{d}=0.75)$. Thus, the powers of alpha, and theta bands are higher in the TD group.

\section{DISCUSSION}

The main aims of the present study were two: a) to examine whether the VWM phases, encoding and retrieval, may be differentially impaired in children with $\mathrm{ADHD}$ and $\mathrm{b}$ ) to define EEG correlates of VWM. In addition to previous studies in ADHD literature, the present work introduces a methodological difference in measuring encoding phase: efficient and inefficient encoding.

With reference to neuropsychological parameters, in the encoding phase we found that the $\mathrm{ADHD}$ groups showed lower duration and frequency of fixation than TD group. As hypothesized, subjects with ADHD look at the stimulus words which appear on the monitor fewer times and for a shorter duration of time than 
the TD group. In accordance with previous studies (Mohammadhasani, Fabio \& Caprì, in press; Mohammadhasani, Fabio, Fardanesh \& Hatami, 2015; Türkan, Amado, Ercan \& Percinel, 2016; Wiegand, 2016), these results indicate that children with $\mathrm{ADHD}$ show an encoding impairment.

With reference to the recalling phase, ADHD groups significantly remembered less words than the TD group. As hypothesized, we found a poorer VWM performance in ADHD groups than TD group. This date indicates that children with ADHD also present a recalling VWM deficit.

The results related to encoding and recalling phases aren't surprising, because it is well-established that the core symptoms of ADHD, defined as inattention, hyperactivity and impulsivity, are associated with WM problems (Burgess et al., 2010; Gau and Shang, 2010; Kofler et al., 2010; Rapport et al., 2008; Rommelse et al., 2008; Toplak et al., 2005).

With regard to neurophysiological parameters, it was found that beta frequency band was higher in the TD group compared to the ADHD groups. It is interesting to note that the beta baseline value was higher in the TD subjects respect to ADHD groups. As explained in the results section, we calculated the differences in beta between baseline and the level during the EE task. The obtained results indicated that $\mathrm{ADHD}$ groups showed an increase in beta during EE task, as TD group, whereas there were still differences in beta band increase when encoding was inefficient.

As regards to alpha and theta frequency bands, these were lower in subjects with ADHD than in TD subjects. This date is in line with previous research reporting a reduced alpha and theta band power in ADHD subjects compared to normal controls (Clarke, Barry, McCarthy, \& Selikowitz, 2001a, 2001b; Clarke et al., 2011; El-Sayed, Larsson, Persson, \& Rydelius, 2002).

The current findings suggest more explanatory directions. Firstly, several studies reporting activation differences in ADHD on WM tasks also reported an absence of significant behavioral differences on the same tasks during the neuroimaging or EGG (Chantiluke, Barrett, Giampietro, Brammer, Simmons, \& Rubia, 2015; Fassbender et al., 2011; Ko et al., 2013; Li et al., 2014; Valera et al., 2005, 2010; Vance et al., 2007). Mattfeld et al. (2016) suggest that one possible explanation for these results is that there is a fundamental heterogeneity among ADHD patients that yields different findings depending upon the proportion of patients with impaired or intact WM represented in any given sample. In other words, these authors assume that the different results aren't due to the verbal or spatial nature of the stimuli during WM tasks, but rather the executive processes invoked by WM conditions that required maintenance and manipulation of larger amounts of information. Consistent with this explanation, our findings demonstrated that when the ADHD groups is adequately activated (i.e. when the encoding is efficient), the increment of the beta band is similar to 
that of the TD group. For these reasons, one future direction of research could be to consider in the analysis the type of encoding in order to check the executive processes involved during WM phases.

Similarly, there is another question related to the present results. As has often been reported, subjects with ADHD can switch from hypo-arousal to hyper-arousal (Shiels \& Hawk, 2010), which is related to the state regulation deficit model (Sergeant, 2005; Van der Mel, Oosterlaan, Heslenfeld, \& Sergeant, 2005); this may explain the alternate phases of efficient and non-efficient encoding. For this reason, in new research it might be important to understand which strategies could contribute to the stabilization of efficient encoding processes and a normalization of arousal.

Thirdly, another possible explanation is that there are sources of variation which are related to both the heterogeneity of subtypes and the methodological heterogeneity. There are two other studies in which EEG signals were recorded while children with ADHD performed a memory test, compared to TD subjects. Lenartowicz et al. (2014) examined the EEG correlates of spatial WM deficits in ADHD during three phases of task: vigilance, encoding and maintenance. Their results indicated an age invariant deficit in vigilance in the ADHD group, supported by reduced alerting responses at fixation and enhanced group differences during encoding and maintenance (for alpha band).

Gomarus, Gomarus, Wijers, Minderaa, \& Althaus (2009) measured eventrelated changes in scalp alpha and theta power while children with ADHD memorized a set of letters for later retrieval. As seen, in contrast to Lenartowicz et al.'s study (2014), no differences in the alpha power band were found in this second study.

It is important to note that there are methodological differences between these two studies. Gomarus, Wijers, Minderaa, \& Althaus (2009) used a verbal WM task and considered children with ADHD-C only, whereas Lenartowicz et al. (2014) employed spatial WM task and included children with combined and inattentive type and children with comorbid ODD in the ADHD group. The results of the aforementioned studies are probably not in agreement because of methodological differences.

At the same time, it seems plausible to assume that the findings of the present study are different from these previous studies, because we used another methodology. Firstly, we employed a VWMT and considered two types of encoding: EE and IE. Secondly, EEG was analysed in three frequency bands: beta, theta and alpha. Thirdly, we recruited three groups: ADHD-I, ADHD-C and a control group. Finally, another reason may be ascribed to the participants of our study who did not have behavioural or emotional disorders in comorbidity. An extension of the sample which includes different groups of subjects with and without comorbidities, belonging to each of the subtypes, 
might give a better understanding of the reasons for the differences found in the different bands.

In conclusion, the present study provides evidence on the context- or statedependency of EEG abnormalities in ADHD during WM task, and, what happens when ADHD subjects are efficiently engaged in a WM task.

\section{CONFLICT OF INTEREST AND AKNOWLEDGMENT}

The authors of the present manuscript certify that they have NO affiliations with or involvement in any organization or entity with any financial interest (such as honoraria; educational grants; participation in speakers' bureaus; membership, employment, consultancies, stock ownership, or other equity interest; and expert testimony or patent-licensing arrangements), or nonfinancial interest (such as personal or professional relationships, affiliations, knowledge or beliefs) in the subject matter or materials discussed in this manuscript. All participants and their parents gave written informed consent. The American Psychological Association's ethical standards were met in the conduct of this study and the Human Ethics Committee of Cognitive Science, Psychological, Educational and Cultural Studies of the University of Messina approved the study protocol. Moreover, the authors thank all the children and their families who agreed to participate in this study.

\section{REFERENCES}

American Psychiatric Association (2013). Diagnostic and statistical manual of mental disorders (5th ed.). Arlington, VA: American Psychiatric Publishing. DC.

Arns, M., Conners, C. K., \& Kraemer, H. C. (2013). A decade of EEG theta/beta ratio research in ADHD: a meta-analysis. Journal of Attention Disorders, $17(5), 374-83$.

Buyck, I., \& Wiersema, J. R. (2015). Electroencephalographic activity before and after cognitive effort in children with attention deficit/hyperactivity disorder. Clinical EEG Neurosciences, 46 (2), 88-93.

Barry, R. J., Clarke, A. R., Johnstone, S. J., \& Brown, C. R. (2009). EEG differences in children between eyes-closed and eyes-open resting conditions. Clinical Neurophysiology, 120 (10), 1806-11. 
Chabot, R. J., \& Serfontein, G. (1996). Quantitative electroencephalographic profiles of children with attention deficit disorder. Biological Psychiatry, 40, 951-963.

Chamberlain, S. R., Robbins, T. W., Winder-Rhodes, S., Müller, U., Sahakian, B. J., Blackwell, A. D., \& Barnett, J. H. (2011). Translational approaches to frontostriatal dysfunction inattention deficit/hyperactivity disorder using a computerized neuropsychological battery. Biological Psychiatry, 69,1192-1203.

Clarke, A. R., Barry, R. J., McCarthy, R., \& Selikowitz, M. (1998). EEG analysis in attention-deficit/hyperactivity disorder: a comparative study of two subtypes. Psychiatry Research, 81,19-29.

Clarke, A. R., Barry, R. J., McCarthy, R., \& Selikowitz, M. (2001a). Electroencephalogram differences in two subtypes of attentiondeficit/hyperactivity disorder. Psychophysiology, 38, 212-221.

Clarke, A. R., Barry, R. J., McCarthy, R., \& Selikowitz, M. (2001b). Age and sex effects in the EEG: differences in two subtypes of attentiondeficit/hyperactivity disorder. Clinical Neurophysiology, 112, 815-826.

Clarke, A. R., Barry, R. J., Dupuy, F. E., Heckel, L. D., McCarthy, R., Selikowitz, M., \& Johnstone, S. J. (2011). Behavioural differences between EEGdefined subgroups of children with Attention-Deficit/Hyperactivity Disorder. Clinical Neurophysiology, 122(7), 1333-41.

Chantiluke, K., Barrett, N., Giampietro, V., Brammer, M., Simmons, A., \& Rubia, K. (2015). Disorder-dissociated effects of fluoxetine on brain function of working memory in attention deficit hyperactivity disorder and autismspectrumdisorder. Psychological Medicine, 45(6), 1195-1205.

Cohen, J. (1988). Statistical power analysis for the behavioral sciences (2nd ed.). Hillsdale, NJ: Lawrence Earlbaum Associates.

El-Sayed, E., Larsson, J. O., Persson, H. E., \& Rydelius, P. A. (2002). Altered cortical activity in children with attention-deficit/hyperactivity disorder during attentional load task. Journal of the American Academy of Child \& Adolescent Psychiatry, 41, 811-819.

Engelhardt, P. E., Nigg, J. T., Carr, L. A., \& Ferreira, F. (2008). Cognitive inhibition and Working memory in attention-deficit/hyperactivity disorder. Journal of Abnormal Psychology, 117, 591-605.

Fabio, R. A. (2017). The study of automatic and controlled processes in ADHD: a reread and a new proposal, Mediterranean Journal of Clinical Psychology, 5, 1, 2-34.

Fabio, R. A., \& Caprì, T. (2015). Autobiographical Memory in ADHD Subtypes. Journal of Intellectual and Developmental Disability, 40, 1-11. 
Fabio, R. A., \& Caprì, T. (2017). The executive functions in a sample of Italian adults with ADHD: attention, response inhibition and planning/organization. Mediterranean Journal of Clinical Psychology, 5 (3), $1-17$.

Fassbender, C., Schweitzer, J.B., Cortes, C.R., Tagamets, M.A., Windsor, T.A., Reeves, G. M., \& Gullapalli, R. (2011). Working memory in attention deficit/hyperactivity disorder is characterized by a lack of specialization of brain function. PLoS One, 6 (11), 27240.

Formaggio, E., Storti, S. F., Avesani, M., Cerini, R., Milanese, F., Gasparini, A., Acler, M., Pozzi Mucelli, R., Fiaschi, A. \& Manganotti, P. (2008). EEG and FMRI coregistration to investigate the cortical oscillatory activities during finger movement. Brain Topography, 21, 100-111.

Geurts, H. M., Verte, S., Oosterlaan, J., Roeyers, H., \& Sergeant, J. A. (2005). ADHD subtypes: do they differ in their executive functioning profile?. Archives of Clinical Neuropsychology, 20, 457-477.

Gomarus, H. K., Wijers, A. A., Mindera, R. B., \& Althaus, M. (2009). Do children with ADHD and/or PDD-NOS differ in reactivity of alpha/theta ERD/ERS to manipulations of cognitive load and stimulus relevance? Clinical Neurophysiology, 120(1), 73-79.

Green, P., Allen, L. M., \& Astner, K. (1996). The Word Memory Test (Manual): A User's Guide to the Oral and Computer-Administered Forms, US Version 1.1. CogniSyst, Inc., Durham, NC.

Guzmán, A., Pereda, E., Mañas, S., Méndez, L. D., Duque, M. R., Almudena, G., \& González, J. J. (2015). The variability of EEG functional connectivity of young ADHD subjects in different resting states. Clinical Neurophysiology, 127, 1321-1330.

Hinshaw, S. P., Carte, E. T., Sami, N., Treuting, J. J., \& Zupan, B. A. (2002). Preadolescent girls with attention-deficit/hyperactivity disorder: II. Neuropsychological performance in relation to subtypes and individual classification. Journal of Consulting and Clinical Psychology, 70, 1099-111.

Ko, C.H., Yen, J. Y., Yen, C. F., Chen, C. S., Lin, W. C., Wang, P. W., \& Liu, G. C. (2013). Brain activation deficit in increased-load working memory tasks among adults with ADHD using fMRI. European Archives of Psychiatry and Clinical Neuroscience, 263 (7), 561-573.

Kamida, A., Shimabayashi, K., Oguri, M., Takamori, T., Ueda, N., Koyanagi, Y., , ... \& Sato, K. (2016). EEG Power Spectrum Analysis in Children with ADHD. Yonago Acta medica, 59,169-173. 
Kuperman, S., Johnson, B., Arndt, S., Lindgren, S., \& Wolraich, M. (1998). Quantitative EEG differences in a nonclinical sample of children with ADHD and undifferentiated ADD. Journal of the American Academy of Child \& Adolescent Psychiatry, 35, 1009-1017.

Lazzaro, I., Gordon, E., Whitmont, S., Plahn M., Li, W., Clarke, S., Dosen, A., Meares, R. (1998). Quantified EEG activity in adolescent attention deficit hyperactivity disorder. Clinical Electroencephalography, 29, 37-42.

Lenartowicz, A., \& Loo, S. K. (2014). Use of EEG to diagnose ADHD. Current Psychiatry Reports, 16(11), 498-517.

Lenartowicz, A., Delorme, A., Walshaw, P. D., Cho, A. L., Bilder, R. M., McGough, J. J., McCracken, J. T., Makeig, S., \& Loo, S. K. (2014). Electroencephalography correlates of spatial working memory deficits in attention deficit/hyperactivity disorder: vigilance, encoding and maintenance. Journal of Neurosciences, 34, 1171-1182.

Liebel, S. W., \& Nelson, J. M. (2017). Auditory and Visual Working Memory Functioning in College Students with Attention-Deficit/Hyperactivity Disorder and/or Learning Disabilities. Archives of Clinical Neuropsychology, 32(8), 980-991.

Li, Y., Li, F., He, N., Guo, L., Huang, X., Lui, S., \& Gong, Q. (2014) . Neural hyperactivity related to working memory in drug-naïve boys with attention deficit hyperactivity disorder. Progress in NeuroPsychopharmacology \& Biological Psychiatry, 53, 116-122.

Liu, Z. X., Glizer, D., Tannock, R., \& Woltering, S. (2015). EEG alpha power during maintenance of information in working memory in adults with ADHD and its plasticity due to working memory training: A randomized controlled trial. Clinical Neurophysiology, 127, 1307-1320.

Loo, S. K., Hale, T. S., Hanada, G., Macion, J., Shrestha, A., McGough, J. J., McCracken, J. T., Nelson, S., \& Smalley, S. L. (2010). Familial clustering and DRD4 effects on electroencephalogram measures in multiplex families with ADHD. Journal of the American Academy of Child \& Adolescent Psychiatry, 29, 368-377.

Loo, S. K., \& Makeig, S. (2012). Clinical utility of EEG in Attention DeficitHyperactivity Disorder: a research update. Neurotherapeutics, 9, 569-587.

Loo, S. K., Cho, A., Hale, T.S., McGough, J., McCracken, J., \& Smalley, S. L. (2013). Characterization of the theta to beta ratio in ADHD: identifying potential sources of heterogeneity. Journal of Attention Disorders, 17(5), 384-92.

Manly, T., Anderson, V., Nimmo-Smith, I., Turner, A., Watson, P., \& Robertson, I. H. (2001). The differential assessment of children's attention: the Test 
of Everyday Attention for Children (TEACH), normative sample and ADHD performance. Journal of Child Psychology and Psychiatry, 42, 1065-1081.

Martínez, A. G., Cortés, J. F., \& Sevilla, J. G. (2018). Implication of Visuospatial and Phonological Working Memory in the Clinical Heterogeneity of AttentionDeficit/Hyperactivity Disorder (ADHD). Annals of Psychology, 34.

Mattfeld, A. T., Whitfield-Gabrieli, S., Biederman, J., Spencer, T., Brown, A., Friedc,R., Gabrieli, J. D. E. (2016). Dissociation of working memory impairments and attention-deficit/hyperactivity disorder in the brain. NeuroImage Clinical, 10, 274-282.

McCarthy, H., Skokauskas, N., \& Frodl, T. (2014). Identifying a consistent pattern of neural function in attention deficit hyperactivity disorder: a meta-analysis. Psychological Medicine, 44, 869-880.

Mohammad, R. M., Khaleghi, A., Nasrabadi, A. M., Rafieivand, S., Begol, M., \& Zarafshan, H. (2016). EEG classification of ADHD and normal children using non-linear features and neural network. Biomedical Engineering Letters, 2, 66-73.

Mohammadhasani, N., Fabio, R. A., Fardanesh, H., \& Hatami, J. (2015). The link between visual attention and memory in ADHD and normally developing students: seeing is remembering?. Reti, saperi, linguaggi, 1, 89102.

Ogrim, G., Kropotov, J., \& Hestad, K. (2012). The quantitative EEG theta/beta ratio in attention deficit/hyperactivity disorder and normal controls: Sensitivity, specificity, and behavioral correlates. Psychiatry Research, 198(3), 482-488.

Sergeant, J. A. (2005). Modeling attention-deficit/hyperactivity disorder: a critical appraisal of the cognitive-energetic model. Biological Psychiatry, 57(11), 1248-1255.

Shiels, K., \& Hawk, L. W. (2010). Self-Regulation in ADHD: The Role of Error Processing. Clinical Psychology Review, 30(8), 951-961.

Snyder, S. M., \& Hall, J. (2006). A meta-analysis of quantitative electroencephalographic power associated with attention-deficit hyperactivity disorder. Journal of Clinical Neurophysiology, 23, 441-456.

Storti, S. F., Formaggio, E., Beltramello, A., Fiaschi, A., \& Manganotti, P. (2010) Wavelet analysis as a tool for investigating movement-related cortical oscillations in EEG-fMRI coregistration. Brain Topography, 23, 46-57.

Türkan, B. N., Amado, S., Ercan, E. S., \& Perçinel, İ. (2016). Comparison of change detection performance and visual search patterns among children 
with/without ADHD: Evidence from eye movements. Research in Developmental Disabilities, 49(50), 205-215.

Valera, E. M., Brown, A., Biederman, J., Faraone, S.V., Makris, N., Monuteaux, M.C., Whitfield-Gabrieli, S., Vitulano, M., Schiller, M., \& Seidman, L.J. (2010). Sex differences in the functional neuroanatomy of working memory in adults with ADHD. American Journal of Psychiatry, 167 (1), 86-94.

Vance, A., Silk, T. J., Casey, M., Rinehart, N. J., Bradshw, J. L., Bellgrove, M. A., \& Cunnington, R. (2007). Right parietal dysfunction in children with attention deficit hyperactivity disorder, combined type: a fucntional MRI study. Molecular Psychiatry, 12(9), 826-832.

van Meel, C., Oosterlaan, J., Heslenfeld, D., \& Sergeant, J. (2005). Motivational effects on motor timing in attention-deficit/hyperactivity disorder. Journal of the American Academy of Child and Adolescent Psychiatry, 44(5), 451-460.

Welch, P. D. (1967). The use of fast Fourier transform for the estimation of power spectra: A method based on time averaging over short, modified periodograms. Audio and Electroacoustics, IEEE Transactions on, 15, 70-73.

Westerberg, H., Hirvikoski, T., Forssberg, H., \& Klingberg, T. (2004). Visuospatial working memory span: a sensitive measure of cognitive deficits in children with ADHD. Child Neuropsychology, 10, 155-161.

Wiegand, I., Hennig-Fast, K., Kilian, Müller, H. J., Töllner, T., Möller, H. J., Engel R. R., Finke, K. (2016). EEG correlates of visual short-term memory as neuro-cognitive endophenotypes of ADHD. Neuropsychologia, 85, 91-99. 
